\title{
Geometry and mechanical function of multijoint extremities from mammals to insects: towards biomimetic design of robotic arm
}

\author{
Oleg Denisov ${ }^{1}$, Natalya Kizilova ${ }^{2,3}$ \\ ${ }^{1}$ Kharkov National University, Ukraine \\ 2 Warsaw University of Technology, Poland \\ ${ }^{3}$ Vilnius Gediminas Technical University, Lithuania \\ E-mails: ${ }^{1}$ biomech@bk.ru, ${ }^{2}$ n.kizilova@gmail.com (correspondingauthor) \\ (Received 26 April 2016; accepted 19 August 2016)
}

\begin{abstract}
Biomechanics of legs of different families of insects is studied. Geometry of different pairs of legs designed for walking, running, swimming, digging and other tasks has been measured on entomological collections. Relationships between the lengths of three main segments (femur, tibia, tarsus) as well as their smaller segments (if any) have been studied. It is shown the cursorial and fossorial legs satisfy the same nondimentional relationships in the insects of different size, habitat and evolutionary age, while the saltatorial, nanatorial and raptoridal legs possess different types of relations. Application of the nature inspired design to engineered macro and micro manipulators and robotic systems is discussed.
\end{abstract}

Keywords: movements of insects, biomechanics of leg, robotics, biomimetic design.

\section{Introduction}

Extremities of animals and human beings are designed as multijoint systems that allows their biomechanical functions like walking, running, jumping or crawling, digging, grasping, manipulation, etc. The number of joints vary from three (shoulder, forearm, hand or hip, shank, foot) in human beings, mammals, etc. to six segments in mites and some other insects. Relationships between the lengths of the segments of human arms, legs and palms have been described by Leonardo da Vinci. For instance, the relative lengths of the human palm segments correspond to the Fibonacci numbers 1, 2, 3, 5, 8 that provides optimal folding in fist according to the Archimedes spiral that allows holding and manipulating of tools.

Dimensionless relations between the segments of insect legs have been studied in connection with insect flight [2] and jumping of fleas, froghoppers and grasshoppers [3, 4], while comparative study of kinematics of the specialized and walking/running legs has not been carried out jet. In this paper the correspondence of design of the extremities (legs) of different families of insects to the optimal multijoint structures is studied accounting for different mechanical abilities of the legs. Such investigations are important for development of multipurpose nature inspired robotic arms.

\section{Methods}

Entomology collections of insects as well as high resolution photos collected in open source museums including the museums of fossils have been studied. The lengths of segments of 3 or 4 pairs of legs depending on the family (Fig. 1a, b) have been measured using freeware image measurement software (ImageJ 1.50i, National Institutes of Health USA (C). In total 21 families of 20 insects in each group have been studied. The measurement data have been used for numerical computations (Excel 2003) of the double ratio value:

$$
W_{j}=\frac{\left(s_{j-1}+s_{j}\right)\left(s_{j}+s_{j+1}\right)}{s_{j}\left(s_{j-1}+s_{j}+s_{j+1}\right)},
$$

(C) 2016 The Authors. Published by VGTU Press. This is an open-access article distributed under the terms of the Creative Commons Attribution License (CC-BY 4.0), which permits unrestricted use, distribution, and reproduction in any medium, provided the original author and source are credited. 
for each three consequent segments $s_{j-1}, s_{j}, s_{j+1}$ of each leg. In that way, n-joint leg is characterized by $\mathrm{n}-2$ values $\mathrm{W}$. The results have been compared to the double ratio value computed for the Fibonacci series $\left\{F_{j}\right\}_{j=1}^{\infty}[1]$ :

$W_{F}=\lim _{j \rightarrow \infty} \frac{\left(F_{j-1}+F_{j}\right)\left(F_{j}+F_{j+1}\right)}{F_{j}\left(F_{j-1}+F_{j}+F_{j+1}\right)} \approx 1.31$.

The results have been separated for different families as well as for the pairs of legs with different functions. Mostly one pair is specified on the function proper to the family while others are used for motion. The insects digging holes, catching preys, gathering food have the first (fore) pair of specialized legs; while the jumping insects and some gathering ones have the last (hind) pair specialized. Besides the fore/hind pairs are also used for eye/wings cleaning and, therefore, used as manipulators.

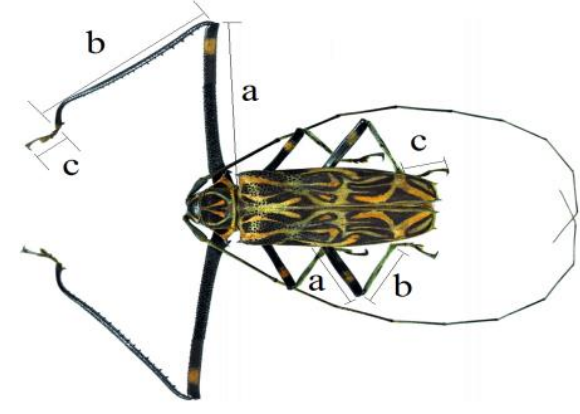

a

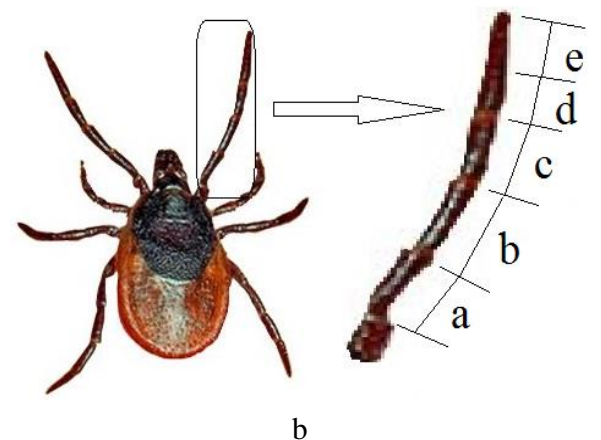

$\mathrm{b}$

Fig. 1. Three segment (a) and five segment (b) insect legs

\section{Results}

Some results summarized on different types of legs and families are presented in Fig. 2. The computed parameters have been averaged over each group. The upper and lower straight lines correspond to the Golden mean ratio 62/38 1.63 and $W_{F} \sim 1.31$.

Contrary to human and mammal arms and palms, the adjacent segments $\mathrm{a}-\mathrm{b}-\mathrm{c}$ (femur, tibia, tarsus) are not related as the Fibonacci numbers. The relations between $a$ and $b$, as well as between $\mathrm{b}$ and $\mathrm{c}$ do not correspond to the Golden ratio which is proper to many optimal biomechanical systems. The only exception is the relation between the tibia and tarsus of the gathering hind legs of bees (Fig. 2). Nevertheless, most of the legs are characterized by $W \sim W_{F}$.

The most optimal in the meaning of the $W$ criteria confirmed on the manipulating arms of mammals and humans, are the running and walking legs (deviations are $0.5 \%$ and $0.7 \%$ accordingly). The digging and gathering legs have the deviations of $2.8 \%$ and $4.4 \%$ that can be considered as minor differences. The grasping and jumping legs differ from the $W_{F}$ design by $11 \%$ and $12.3 \%$ accordingly, which is also not large for the biological data. The swimming hands have special design defined by $W$ values which are in $27 \%$ higher than the $W_{F}$ value. In the most cases the segments are designed in the way allowing perfect folding of the leg into a chain (for the case presented in Fig. 1a) or spiral (Fig. 1b).

The lengths of the segments corresponded to the measurement data averaged over the family. The moments of forces $m_{j}$ produced in the joints have been accepted to be proportional to the lengths $l_{j}$ of the corresponding segments. Dynamics of folding of the leg when the beginning of the first segment a (shoulder) is fixed and the end of the last segment $\mathrm{c}$ or e (tips of the claws) reaches the shoulder. As optimization criteria, the energy $E_{m}$ spent for the movement and the 
operational time $t_{0}$ have been considered. The optimal Pareto solutions have been obtained for each case. The results are presented in Fig. 3 in dimensionless form. It was shown, the relations $l_{j-1} / l_{j}$ and $l_{j} / l_{j+1}$ are mostly far from 62/38 (red line in Fig. 2), but close to $W_{F}$ value (blue line in Fig. 2).

The walking and running legs were found the most optimal in the meaning of the pair of criteria $\left(E_{m}, t_{0}\right)$ (Fig. 3). The digging legs are the slowest in performance but optimal for energy consumption. The jumping legs are the fastest but energy consuming. In some areas the families of frontiers are located very close that means optimal performance for different types of action like digging and swimming and running, gathering and grasping (Fig. 3).

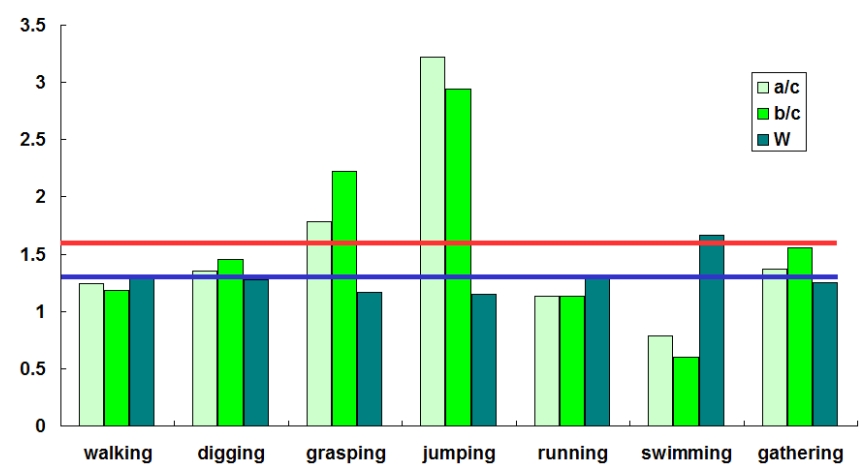

Fig. 2. Distribution of the $\mathrm{a} / \mathrm{c}, \mathrm{b} / \mathrm{c}$ and $W$ values for insect legs of different function

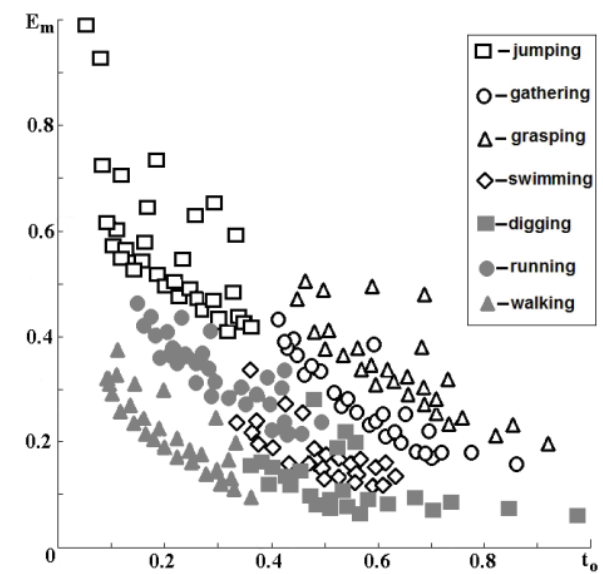

Fig. 3. Multi-objective Pareto frontiers for $E_{m}$ and $t_{0}$

\section{Conclusions}

Geometry of insect legs as mechanical multi-link manipulators has been studied. The measurement data revealed high proximity of the running and walking legs, as well as digging and gathering legs to the double ratio $W_{F}$ proper to upper extremities of humans and some animals, while the grasping, jumping and swimming legs are further from this value. Analysis of dynamics of the n-link leg revealed the relationships between the lengths of the links that are needed for optimal performance, namely the maximal force generation at the end of the leg at the same efficiency of the muscles rotating the joints. The perfect folding of the leg into a chain/spiral confirms the dynamical ability of the leg with the same lengths of the links. The obtained relationships are useful for designing multipurpose robotic arms and legs. Usually the robotic arms 
are designed basing on one optimization criteria such as energy consumption, performance time, simplicity of the control [5], while the proposed approach allows multicriteria optimization for a pair of optimal criteria which are most suitable for the designed robotic system. The results for some other pair of the criteria will be presented in the full-text paper.

\section{References}

[1] Petukhov, S. V. 1981. Biomechanics, bionics and symmetry. Moscow: Nauka. 238 p.

[2] Dudley, R. 2000. The biomechanics of insect flight: form, function, evolution. Princeton: Princeton University Press. 476 p.

[3] Ritzmann, R. E.; Zill, S. N. 2009. Walking and jumping, in Encyclopedia of insects. Ed. by V. H. Resh, R. T. Cardé. San Diego: Academic Press, 1044-1048.

[4] Casas, J.; Simpson, S. J. [eds.]. 2008. Advances in insect physiology, vol. 34: insect mechanics and control. San Diego: Academic Press. 379 p.

[5] Corke, P. 2011. Robotics, vision and control: fundamental algorithms in MATLAB. Berlin Heidelberg: Springer-Verlag. 495 p. http://dx.doi.org/10.1007/978-3-642-20144-8 